\title{
DIAGNOSTICS OF SUBPICOSECOND ELECTRON PULSE BY THE FLUCTUATION METHOD
}

\author{
T. Watanabe ${ }^{1}$, K. Nakamura, T. Ueda, M. Uesaka, Univ. of Tokyo, Ibaraki, Japan
}

\begin{abstract}
We performed the diagnostics of subpicosecond and picosecond electron pulses by the fluctuation method. The method is based on the observation of the shot-drivennoise fluctuations of incoherent radiation and was proposed by $\mathrm{M}$. Zolotorev et al. [1]. In our case, Cherenkov radiation and optical transition radiation emitted from the electron pulse were detected by the photodiode for the time-domain measurement and by the spectrometer for the frequency-domain measurement. Results by these measurements were compared with the pulse duration independently taken by the femtosecond streak camera. Another feature of our diagnostics is the large transverse beam size. The effect of the transverse beam size upon the fluctuation was discussed with the aid of the numerical simulations.
\end{abstract}

\section{NUCLEAR ENGINEERING RESEARCH LABORATORY, UNIV. OF TOKYO}

At Nuclear Engineering Research Laboratory (NERL), University of Tokyo, it is now possible to generate several hundreds femtosecond (Full Width at Half Maximum) electron single pulse from $18 \mathrm{MeV}$ linac that consists of a laser photocathode $\mathrm{rf}$ gun and a chicane-type magnetic pulse compressor, and from $35 \mathrm{MeV}$ linac that consists of a thermionic gun and an arc-type magnetic pulse compressor [2]. The plasma cathode experiment, which is supposed to generate tens of fs electron pulse, is under way [3] . Concerning the diagnostics of short electron pulses, the femtosecond streak camera (FESCA-200, Hamamatsu Photonics), of which time resolution is 200 $\mathrm{fs}$, has been used [4]. Since the shortest electron pulse would beyond the time resolution in near future, it is necessary to construct an alternative diagnostic method. For the purpose, we have focused our attention on diagnostics by the coherent transition/diffraction radiation (CTR/CDR) interferometer and the CTR/CDR polychromator so far $[5,6]$. Although fine agreements have been obtained by these methods, we adopt one more technique; the fluctuation method. Because to compare measurement results by several methods is presumably important in order to make sure the reliability of each method.

\section{FLUCTUATION METHOD}

The spectrum of radiation $I(v)$ emitted by the electron

\footnotetext{
†wata@tokai.t.u-tokyo.ac.jp
}

pulse, such as transition radiation and Cherenkov radiation, can be written as $[5,6]$,

$$
I(v)=N[1+(N-1) f(v)] I_{0}(v),
$$

where $N$ is the number of electrons in a bunch, $I_{0}(v)$ is the spectrum emitted by a single electron, and $f(v)$ is the bunch form factor that corresponds to the Fourier transform of the distribution function of the electron bunch. The first tem in Eq. (1) expresses the incoherent radiation and the enhancement of the radiation intensity that can be found in the second term occurs from the coherent effect. When the wavelength of the radiation is longer than the bunch duration, the electric fields of the radiation that is emitted by each electron are increased as the coherent radiation, of which intensity is proportional to $N^{2}$. When the wavelength of the radiation is shorter than the bunch duration, the large random argument in the phase of the radiation emitted from a single electron, $E_{0}(t)$, causes the total electric field $E(t)$ to randomly fluctuate in a bunch [7];

$$
E(t)=\sum_{j}^{N} E_{0}\left(t-t_{j}\right) \text {. }
$$

An existing photodiode can measure only the timeintegrated intensity $\int|E(t)|^{2} d t$ and eventually shot-byshot fluctuations in the radiation is obtained in the experiment. As proposed in Ref. [1], the fluctuation of the time-integrated intensity gives the information of the number of independent parts in a bunch, $N_{\text {indep }}$;

$$
\sigma^{2}=\frac{F}{N_{\text {indep }}}=\frac{F \tau_{c o h}}{\tau_{b}},
$$

where $F$ is a form factor that is computed from the Fourier transform of the bunch distribution, and $\tau_{c o h}, \tau_{b}$ are the duration of the independent part and the bunch, respectively. The duration of the independent part, $\tau_{c o h}$, which is associated to the coherent time, results from the finite length of the envelope of the electric field emitted by a single electron and therefore it can be calculated by knowing the bandwidth of the radiation.

As well as the fluctuations in the time domain, one can see the radiation fluctuating in the frequency domain. Moreover, it is known that the measurement in the frequency domain provides more details. Observing the spike width of the spectrum, the fluctuations of the bunch duration itself can be obtained from shot to shot, since the spikes have a characteristic width $\Delta \omega \sim \tau_{b}^{-1}$. The pedestal of the spectrum gives the number of transverse 
modes. Further, the longitudinal bunch distribution is deduced by the statistical analysis of the fluctuation. After accumulation of the Fourier transform $\Gamma(\tau)$ of the spectrum among hundreds of bunches, the variance of the fluctuations of $\Gamma(\tau)$ is proportional to the convolution of the bunch profile $I(t)$ as follows;

$$
\left\langle|\Gamma(\tau)-\langle\Gamma(\tau)\rangle|^{2}\right\rangle \propto \int d t I(t) I(t-\tau)
$$

where the angular brackets denote ensemble average.

It is worth noticing that throughout the theory, it is assumed that the quantum fluctuations of the number of photons in the detector are negligible.

\section{NUMERICAL ANALYSIS}

In the Time-domain measurement, where the fluctuations of the time-integrated intensity is detected, it is necessary to distinguish the fluctuations of the bunch parameters, such as the electron charge and the bunch distribution, and those coming from the statistical properties as mentioned in Chap. 2. For the purpose, the expectations of the fluctuation at several pulse durations were computed as shown in Fig. 1. The spectrum of the radiation is limited so that the central wavelength is 500 $\mathrm{nm}$ and the bandwidth is $5 \mathrm{~nm}$. One can easily imagine that as the bunch becomes shorter, it is easier to distinguish the fluctuations from the noise. If the noise is around $30 \%$, for instance, less than $500 \mathrm{fs}$ is required. You note that the noise from the fluctuations of the electron charge can be calibrated by observing it. Further, actual fluctuation is enhanced with the narrower band pass filter. However the narrow band pass filter suppress the light intensity.

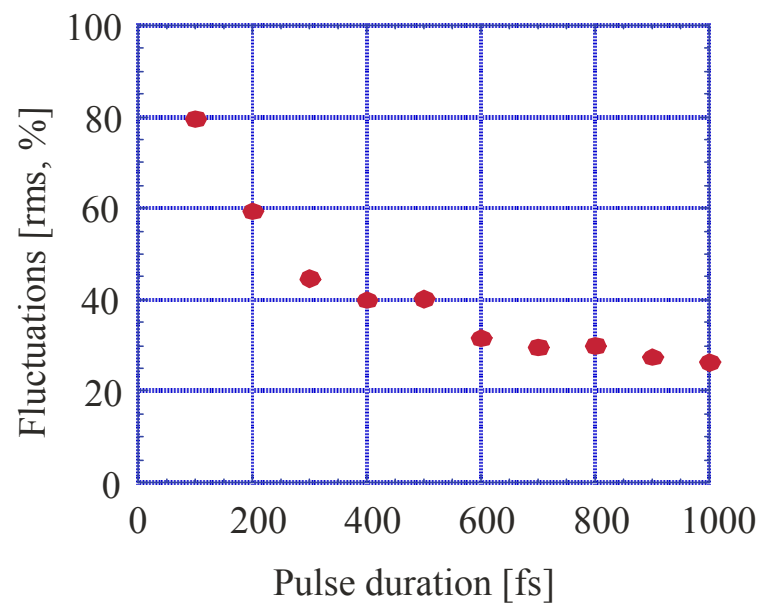

Figure 1: Fluctuations at several pulse durations.

Figure 2 represents the spectrum of the incoherent radiation that was estimated under the condition of the following parameters; the pulse duration is $1.0 \mathrm{ps}$, the distribution is Gaussian and the number of transverse modes is 5 . The spike width is proportional to the inverse of the bunch duration (cf. Chap. 2). The pedestal of the spectrum comes from the finite transverse emittance [7]. It is worth noticing that both Fig. 1 was estimated under the assumption of infinitesimal diameter. If the transverse emittance is not negligible, the fluctuation is restrained. The bunch, of which duration is $5.0 \mathrm{ps}$ with infinitesimal diameter, exhibits similar fluctuations by the 1.0-ps long 5 -independent modes bunch.

In Figs. 1 and 2, one can see one of characteristics of the method that the shorter pulse can be measured easier, which is not true in most other diagnostic methods.

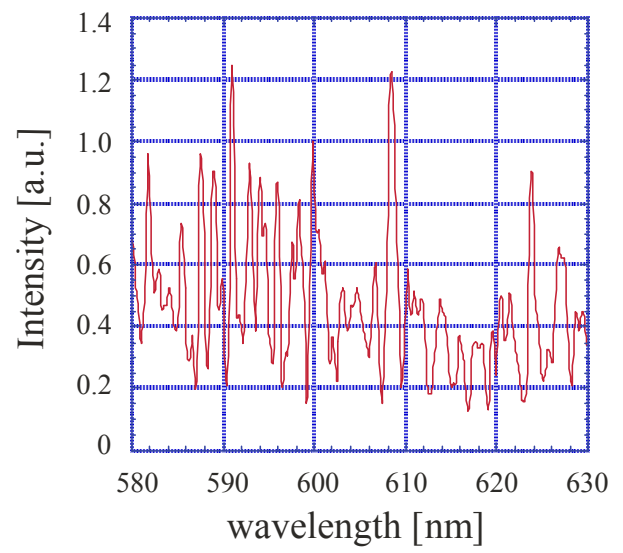

Figure 2: Spectrum of the radiation.

\section{EXPERIMENT}

First, the time-integrated intensity of the incoherent radiation was measured as the function of the bandwidth. The experimental setup is illustrated in Fig. 3. When an electron pulse goes through in air, Cherenkov light is emitted. After that, when the electron pulse passed through the $\mathrm{Al}$ foil, transition radiation is emitted. We measured both radiation but the intensity of optical transition radiation was too low to detect. Therefore through the experiment, Cherenkov radiation was detected.

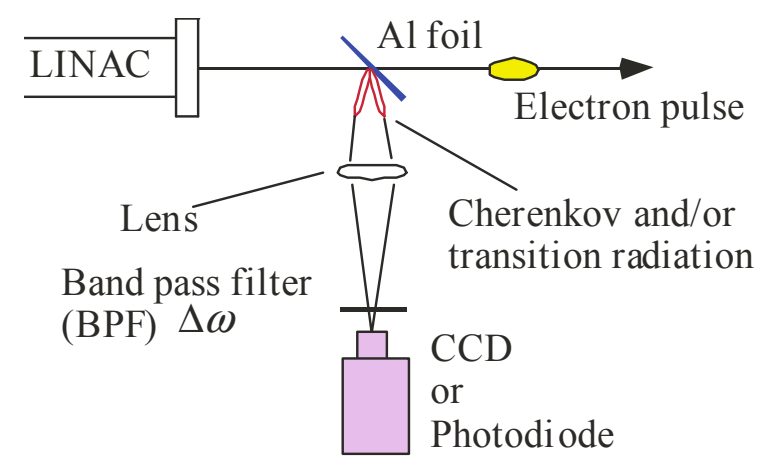

Figure 3: Experimental setup

In the experiment, the spectrum is limited by the band pass filter. Figure 4 shows the fluctuations of timeintegrated intensity that was taken by the photodiode, in which 1.0 and 1.5 ps electron bunch respectively from 18 and $35 \mathrm{MeV}$ linacs were measured. The bunch durations were decided by the independent measurement by the streak camera. In the case of 1.0 ps bunch, the 
experimental data of the fluctuations, which is plotted in Fig. 4 (a), was estimated to be 4.5 ps. In the same way, $1.5 \mathrm{ps}$ bunch was measured to be as long as $30 \mathrm{ps}$ from the fluctuations. These discrepancies were due to the transverse emittance of the bunch (cf. Chap. 3). At the exit of 18 and $35 \mathrm{MeV}$ linacs, the transverse beam sizes are measured to be about 3 and $5 \mathrm{~mm}$ (FWHM of Gaussian distribution), respectively. The emittance of the $18 \mathrm{MeV}$ linac is smaller than that of $35 \mathrm{MeV}$, since 18 $\mathrm{MeV}$ linac consists of a laser photocathode rf gun. Therefore we guess that the discrepancy in the case of 35 $\mathrm{MeV}$ linac were larger compared with $18 \mathrm{MeV}$ linac. If the number of transverse modes $\mathrm{q}$ is estimated with $a$ priori information on the bunch duration from the streak camera measurement, $\mathrm{q}$ is 4.5 (18 MeV linac ) and 20 (35 Mev linac).

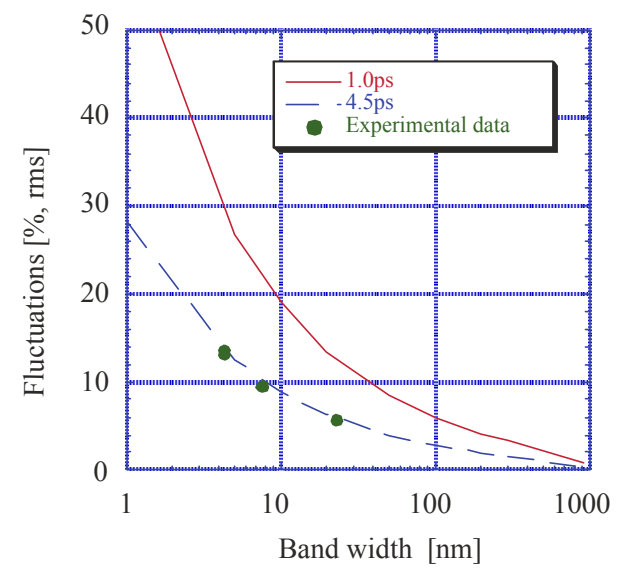

(a) $18 \mathrm{MeV}$ linac

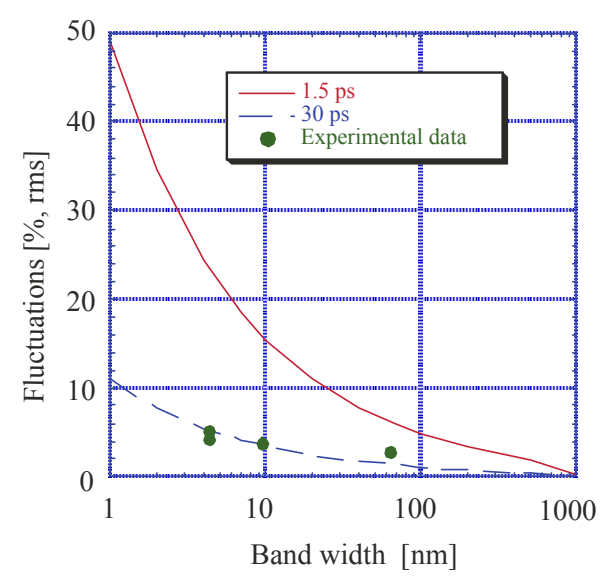

(b) $35 \mathrm{MeV}$ linac

Fig. 4 Time-domain measurement. Dots are the experimental data. Solid lines are fluctuations estimated from the bunch duration taken by the streak camera.
The measurement of the fluctuation in the spectrum is under way at $18 \mathrm{MeV}$ linac. From the time-domain measuremnt, the number of transverse modes is around 5, which was the condition of the numerical simulation (cf. Fig. 2). Therefore it is expected that the spectrum that is shown in Fig. 2 is obtained in the experiment, although it is not exactly same but similar.

\section{SUMMARY}

Numerical simulations on the time-domain and the frequency-domain measurement of the fluctuations were performed. It was shown that both in the time-domain and the frequency-domain measurement, the short pulse can be measured easily. Experiments were carried out in the time-domain measurement. Although there were large discrepancies between the fluctuation method and the femtosecond streak camera, it is reasonable if the transverse emittance is considered. We plan to resolve the fluctuation in the frequency domain and deduce the bunch distribution in near future.

\section{ACKNOLEDGEMENTS}

We acknowledge useful discussions with M.S. Zolotorev and P. Catravas.

\section{REFERENCES}

[1] M.S. Zolotorev and G.V. Stupakov, SLAC-PUB 07132, 1996; in Proceedings of the 1997 Particle Accelerator Conference (PAC'97), Vancouver, Canada (1998) 2180.

[2] M. Uesaka et al., Phys. Rev. E 54 (1994) 3068.

[3] H. Nasr et al., Nucl. Instrum. Meth. A, 455 (2000) 149.

[4] M. Uesaka and T. Watanabe et al., J. Nucl. Mater. 248 (1997) 380.

[5] T. Watanabe et al., Nucl. Instrum. Meth. A, 437 (1999) 1.

[6] T. Watanabe et al., Nucl. Instrum. Meth. A, in press.

[7] P. Catravas et al., Phys. Rev. Lett. 82 (1999) 5261. 